\title{
Ventricular tachycardia: beginning and ending fate of a benign invasive cardiac lipoma
}

\author{
Olga Vriz ${ }^{1,2}$, Mawada M. Ahmed ${ }^{3}$, Suleiman Kharabsheh ${ }^{1}$, Mohammed Aladmawi', Bandar Alamro1, \\ Afrah AlSomali ${ }^{2}$, Nader Fawzy ${ }^{2}$, Aly Alsanei ${ }^{1}$, Najmeddine M. Echahidi ${ }^{1}$ \\ ${ }^{1}$ Heart Centre Department, King Faisal Specialist Hospital and Research Center, Riyadh; ${ }^{2}$ School of Medicine, Alfaisal \\ University, Riyadh; ${ }^{3}$ Security Forces Hospital, Cardiology Department, Riyadh, Saudi Arabia
}

\begin{abstract}
Cardiac lipomas are rare benign heart tumors. Their clinical manifestations primarily depend on their dimension and location. We describe a case of a 14-year-old boy complaining of palpitations. ECG showed non-sustained ventricular tachycardia (NSVT). Echocardiography and cardiac MRI showed a large apical mass suggestive of a cardiac lipoma. The patient underwent open-heart surgery which detected a pericardial mass, partially attached to the myocardium, and crossed by the distal segment of LAD. This critical anatomical relationship prevents its complete resection. The biopsy confirmed mature fat tissue. Postoperatively, Propranolol was started for NSVT episodes. After 8 months, he presented with VT recurrence. A subcutaneous ICD (S-ICD) was implanted. Two months later, he was admitted for an electrical storm with six appropriate shocks. Since then, amiodarone was added. Lipomas are not always benign and their sur-
\end{abstract}

Correspondence: Mawada Ahmed, Cardiology Department, Security Forces Hospital, P.O. Box 3643, Riyadh 11481, Saudi Arabia. Tel. +966.11.8026731 - Fax: +966.11.8024321.

E-mail: mawadamoh@hotmail.com

Key word: Ventricular tachycardia; cardiac tumor; lipoma; ablation.

Contributions: OV and MA contribute equally and share first authorship, other authors share in discussion and images interpretation. All authors have read and approved the final version of the manuscript.

Consent for publication: Written informed consent was obtained from the patient's father for publication of this case report and any accompanying images.

Conflict of interest: The authors declare no conflict of interest.

Received for publication: 26 March 2020.

Accepted for publication: 7 May 2020.

${ }^{\circ}$ Copyright: the Author(s), 2020

Licensee PAGEPress, Italy

Monaldi Archives for Chest Disease 2020; 90:1288

doi: 10.4081 monaldi.2020.1288

This article is distributed under the terms of the Creative Commons Attribution Noncommercial License (by-nc 4.0) which permits any noncommercial use, distribution, and reproduction in any medium, provided the original author(s) and source are credited. gery might be very difficult and unhelpful. A multidisciplinary team is crucial for their diagnosis and management.

\section{Introduction}

Cardiac lipomas are encapsulated tumors of the heart composed primarily of mature fat cells and represent $8.4 \%$ of the all benign tumors. Lipomas are usually asymptomatic. However, arrhythmias and symptoms of compression can occur depending on the location of the tumor [1]. Typical locations are the endocardium ( $50 \%$ of cases) of the right atrium and left ventricle (LV), in $25 \%$ of cases the myocardium can be involved and another quarter located sub-epicardially [2].

\section{Case Report}

A 14-year-old male presented to the Emergency Room (ER) for prolonged palpitations, started 1 year back, characterized by a brief period of palpitations. Over time these episodes became more frequent and prolonged. He denied other symptoms.

In ER he was hemodynamically stable: Heart rate $76 \mathrm{bpm}$ in sinus rhythm, blood pressure was $101 / 54 \mathrm{mmHg}$. A physical examination and labs were unremarkable. On the other hand, Electrocardiogram (ECG) showed a run of non-sustained ventricular tachycardia (NSVT) and premature ventricular complexes (PVCs). Also, there were diffuse ST elevation changes mainly seen in the inferolateral leads likely due to pericardial irritation from the tumor (Figure 1 A1,A2). The morphology of NSVT and PVCs were highly suggestive of right ventricular (RV) origin from the inferior apical region. The transthoracic echocardiography (TTE) showed an echogenic mass mainly at the apex of LV and anterolateral wall, otherwise normal heart. Cardiac magnetic resonance (MRI) showed a large solid mass in the pericardial space, at the level of LV and RV apex, measuring $7.8 \times 5.3 \mathrm{~cm}$. The lipoma was causing mass effect with high signal intensity on T1 and T2weighted imaging, with complete signal drop-out using fat-saturation imaging (Figure 2A). No clear cleavage line with the myocardium. Cardiac CT showed the lipoma crossed by distal left anterior descending (LAD) coronary artery (Figure 2B). Coronary angiogram revealed normal coronary arteries (Figure 2C).

Since the mass was likely a big lipoma and because of the VT, heart team decided to remove it surgically. The mass was found to occupy almost $2 / 3$ of the left ventricle going to the apex and involving part of the right ventricle. The lipoma was encapsulated 
within the epicardium on one side, but there was no cleavage line on the myocardial side (Figure $3 \mathrm{~A}, \mathrm{~B}$ ). The LAD was running on the lateral side of the mass, one small distal branch was feeding the lipoma and was resected knowing that could be responsible for some degree of localized myocardial ischemia (Figure 3C). Although a significant part of the lipoma was removed (Figure 3C), a considerable part of it remained in situ.

During the hospital admission, the patient had several episodes of NSVT with two different morphologies: one identical to the initial morphology before surgery (Figure $1 \mathrm{~B} 1$ ) and the second morphology showed an aspect of RBBB suggestive of LV origin from the infero-apical region (Figure 1 B2). The alternate LBBB and RBBB aspects were related to the mass location. Cardiac MRI was repeated before discharge and documented large residual apical LV mass (Figure 3D). Histopathology of the excised mass showed mature adipose tissue confirming to be a lipoma (Figure 3D). Bblockers were started in the form of propranolol and the patient was discharged after 7 days.

The patient remained asymptomatic for 8 months then he was admitted with evidence of NSVT. On ECG sustained VT was similar to the VT recorded previously. TTE showed residual LV apical mass. MRI confirmed the presence of the lipoma and showed a subendocardial late enhancement at the apical segment of the interventricular septum (Figure 2E), which was a new finding consistent with non-transmural infarction, likely related to the injured distal LAD during surgery. Coronary angiography was repeated showing a distal occlusion of a small size LAD branch (Figure 2F), not suitable for intervention. No Q wave on ECG or wall motion abnormality on TTE. The case was discussed in a heart team meeting and there was an agreement for subcutaneous ICD (S-ICD) implantation along with medical therapy and close follow-up.

After 2 months, he came back for palpitations and electrical storm. Interrogation of S-ICD showed that six appropriate shocks were delivered in 2 days for very fast VT. The patient's medical therapy was implemented with amiodarone. No endocardial ablation of the ventricular arrhythmia appears to be indicated since the trigger is still present as a residual mass in the pericardial space. Moreover, it would have been extremely difficult and dangerous to attempt epicardial radiofrequency catheter ablation of VT in a post-surgical pericardium space where the mass may prevent reaching the foci. Furthermore might be located in close vicinity to the distal segment of LAD. However, if the patient showed up with frequent and refractory VTs, still such complex ablation could be proposed.

Since then, the patient remained asymptomatic and no further arrhythmia was recorded in the memory of S-ICD.

\section{Discussion}

Among cardiac tumors, which are extremely rare $(0.001-0.03 \%$ in most autopsy cases) lipomas are the second most common benign
A1

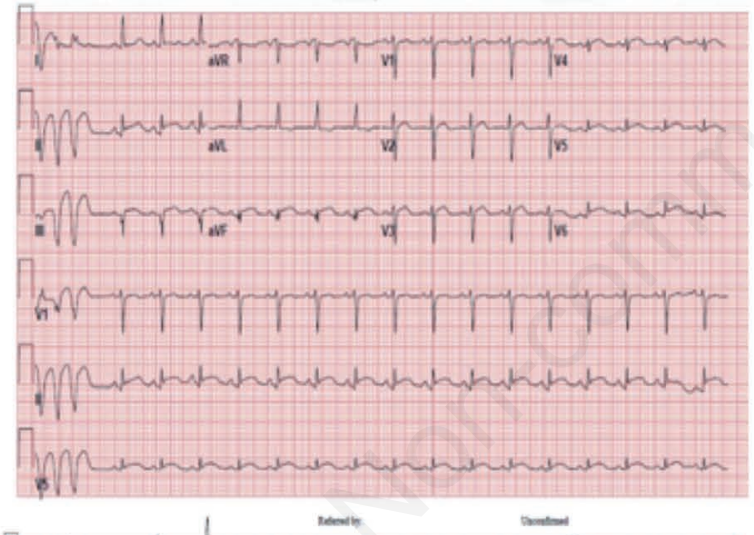

B1

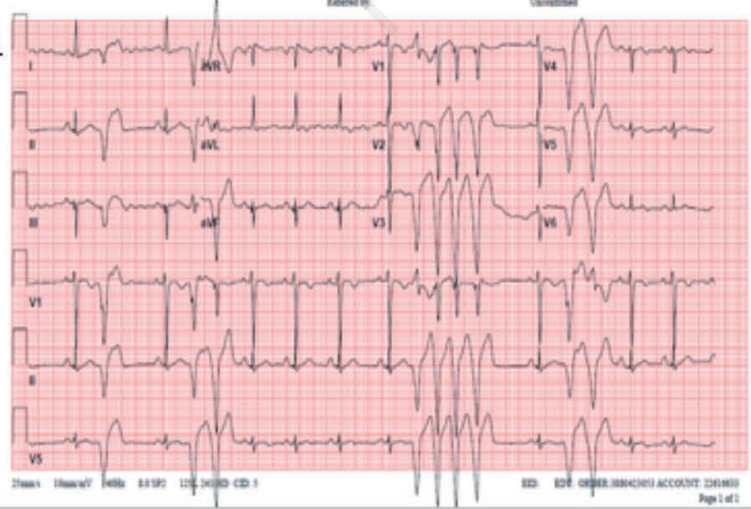

A2

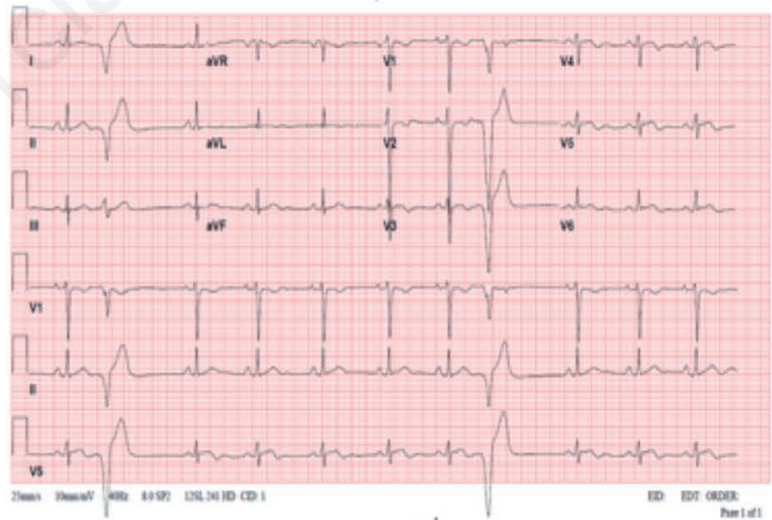

B2

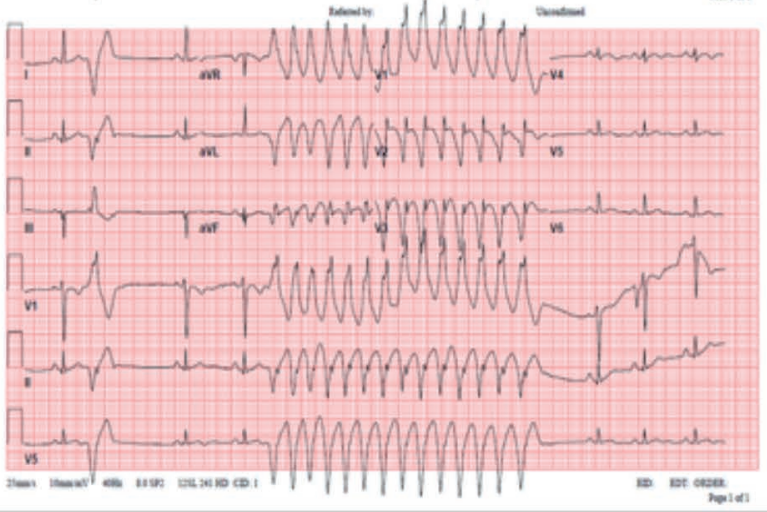

Figure 1. A) ECG at first admission, pre-surgery; A1 showed a run of NSVT and diffuse ST segment elevation constant with pericarditis, A2 showed PVCs, the morphology of NSVT and PVCs was highly suggestive RV origin. B) ECG after surgery; B1 ECG showed NSVT of LBBB morphology suggestive of RV origin while B2 showed NSVT of RBBB morphology suggestive of LV origin when the patient was admitted to the hospital for palpitation after 8 months of surgery. ECG, electrocardiogram; LBBB, left bundle branch block; LV, left ventricular; NSVT, non-sustain ventricular tachycardia; PVCs, premature ventricular complexes; RV, right ventricular. 
tumor after myxomas with a reported incidence of about 10-19\% [1]. Lipomas are made by adipose tissue, it can be localized anywhere in the heart most frequently they arise from endocardium (50\%), epicardium (25\%), and even intramyocardial (25\%) [2]. The most common chambers involved are left ventricle and right atrium [3]. According to the literature, lipoma is typically seen in fifth and sixth decades of life, but any age group can be affected and both genders are equally involved. The etiology of lipomas remains unknown. An association with the chromosome 12 gene re-arrangements has been reported in solitary lipoma cases where an abnormality in the HMGA2-LPP fusion gene was documented [4].

They are extremely variable in size. Most of the time they are asymptomatic but when symptoms are present, they are nonspecific and typically are related to the size and location of the tumor such as fatigue, dyspnea, palpitation, syncope and chest pain [4]. Ventricular tachycardia is caused either by damage to the myocardium or by the involvement of the conduction system. Sudden death also was reported [5]. Lipomas tend to grow and for that reason, they require periodic echocardiographic follow-up. Echocardiography is the initial imaging method. Lipomas usually broad-based, immobile, without a pedicle and well circumscribed, although this is not a strict characteristic. Cardiac MRI remains the gold standard investigation to diagnose lipomas and for better assessment of the tumor proximity to adjacent structures. Typically, on cardiac MRI lipoma has a homogenous high signal intensity in both T1- and T2-weighted images when compared with myocardium [6]. Coronary arteriography can provide valuable information for the surgeon by defining the primary anatomy. Sometimes we need a biopsy before the intervention to differentiate lipoma from well-differentiated liposarcomas.

Surgical excision is ordinarily the therapeutic intervention of choice in symptomatic patients (arrhythmia or signs of compression), and usually they are easily resected. However, since the tumor is relatively rare there is no strong experience for optimal timing of intervention in asymptomatic patients [7]. The tumor usually can be easily detached but sometimes the separation can be challenging because it can be immersed in the LV wall, can involve the conduction system, coronary arteries as it was in our case, valves and chordae. The rate of lipoma recurrence after total and subtotal resection is extremely low.
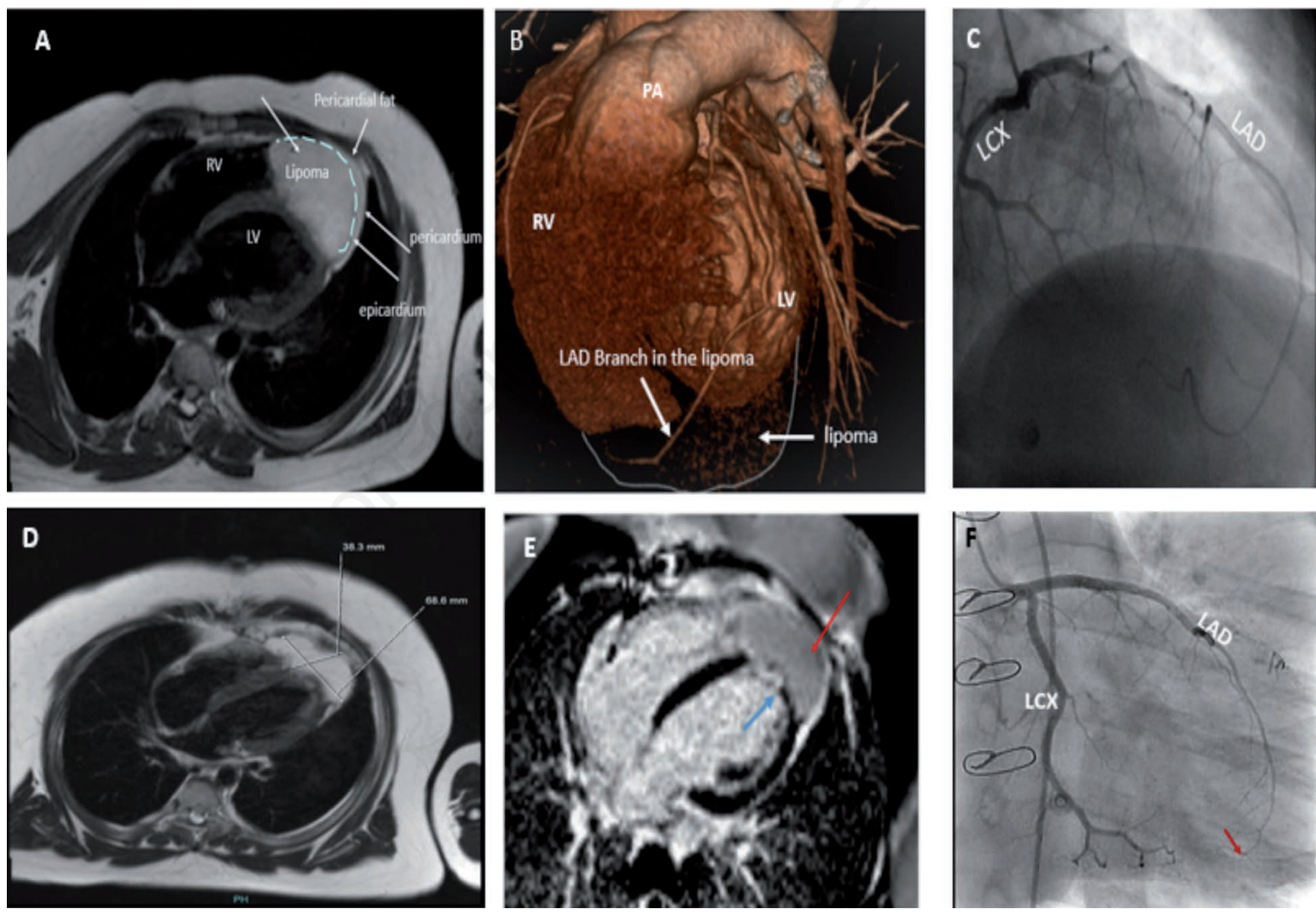

Figure 2. A) MRI. Dark Blood HASTE imaging that shows isointense signal of the mass; the lipoma extends from the LV to the RV; in light blue can be seen the pericardium that encapsulates the lipoma and divide it from the pericardial fat. B) Cardiac CT, 3D reconstruction showed the LAD in relation to the lipoma and the feeding branch. C) Coronary angiography showed normal LAD and LCX before surgery. D) MRI after surgery demonstrated the residual part of lipoma. E) Delayed-enhancement MRI after 8 month of surgery demonstrated small apical infarction (blue arrow) and the residual lipoma (red arrow). F) Coronary angiography after surgery that demonstrated the distally occluded LAD (red arrow). CT, computed tomography; L, left; LAD, left anterior descending; LCX, left circumflex; LV, left ventricle; MRI, magnetic resonance image; $R V$, right ventricle. 

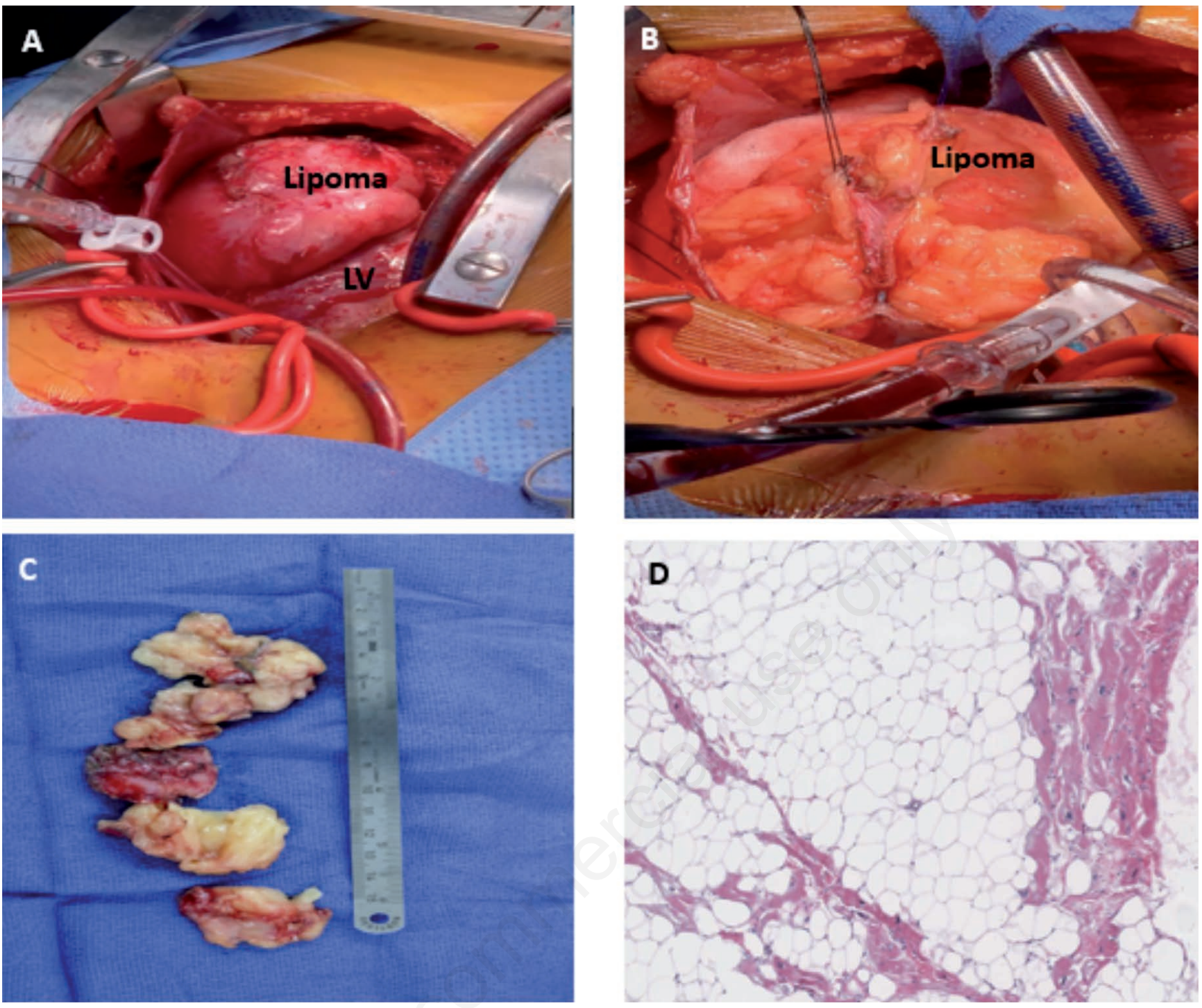

Figure 3. A) Showed lipoma in relation to the left ventricle (LV) before excision. B) The surgical incision of the lipoma. C) Surgical specimens after resection. D) Histopathology of the tumor, the lipoma specimen reveals clearly mature adipocytes.

Our case represents the cluster of rare characteristics of lipoma: i) adolescent patient; ii) a big mass occupying involving part of the LV and RV apex; iii) involvement of LAD and likely the conduction system; iv) no clear cleavage section between the mass and the myocardium and for this reason the tumor was incompletely removed; v) in terms of symptoms the tumor was responsible for extremely fast VT; vi) surgical resection, in this case, didn't change the clinical picture or the prognosis of the patient.

Our case delivered several messages: 1) A multidisciplinary team is of significant importance for the diagnosis and management of this disease. 2) Lipomas should not be considered always as a benign tumor since it might lead to refractory and life-threatening ventricular arrhythmias. 3) Cardiac surgery for such tumor is not all the time curative therapy since the tumor may display a complex anatomical relationship with the neighborhood structures (sometimes vulnerable). 4) Radiofrequency catheter ablation may have significant limitations when it comes ablating within the epicardium in close vicinity of epicardial coronary arteries.

\section{Conclusions}

Cardiac lipomas are rare benign tumors with no guidelines available for their management that should be multidisciplinary. Surgery is recommended when there are symptoms but complete resection is not always feasible. In this case, although surgery was supposed to be the best option for arrhythmia therapy, it turned out to be useless and radiofrequency ablation is too risky that might end up in unsatisfactory results.

\section{References}

1. Girrbach F, Mohr FW, Misfeld M. Epicardial lipoma-a rare differential diagnosis in cardiovascular medicine. Eur J Cardiothorac Surg 2012;41:699-701. 
2. Ismail I, Al-Khafaji K, Monica Mutyala M, et al. Cardiac lipoma. J Community Hosp Intern Med Perspect 2015; 5:28449.

D'Souza J, Shah R, Abbass A, et al. Invasive cardiac lipoma: a case report and review of literature. BMC Cardiovasc Disord 2017; 17:28.

3. Italiano A, Ebran N, Attias R, et al. NFIB rearrangement in superficial, retro- peritoneal, and colonic lipomas with aberrations involving chromosome band 9p22. Genes Chromosomes Cancer 2008;47:971-7.

4. Jayaprakash S. Clinical presentations, diagnosis, and manage- ment of arrhythmias associated with cardiac tumors. J Arrhythm 2018;34:384-93.

5. Arslan S, Gundogdu F, Acikel M, Kantarci AM. Asymptomatic cardiac lipoma originating from the interventricular septum diagnosed by multi $\square$ slice computed tomography. Int J Cardiovasc Imaging 2007;23:277 $\square 9$

6. Bagwan IN, Sheppard MN. Cardiac lipoma causing sudden cardiac death. Eur J Cardiothorac Surg 2009;35:727.

7. Korantzopoulos P, Patsouras D, Kountouris E, et al. Lipoma of the interatrial septum in a patient with chronic atrial fibrillation: A case report. Angiology 2006;57:513-6. 